\title{
Character of states near the Fermi level in (Ga,Mn)As: Impurity to valence band crossover
}

\author{
T. Jungwirth, ${ }^{1,2}$ Jairo Sinova, ${ }^{3}$ A. H. MacDonald, ${ }^{4}$ B. L. Gallagher, ${ }^{2}$ V. Novák, ${ }^{1}$ K. W. Edmonds, ${ }^{2}$ A. W. Rushforth, ${ }^{2}$ \\ R. P. Campion, ${ }^{2}$ C. T. Foxon, ${ }^{2}$ L. Eaves,${ }^{2}$ E. Olejník, ${ }^{1}$ J. Mašek, ${ }^{5}$ S.-R. Eric Yang, ${ }^{6}$ J. Wunderlich, ${ }^{7}$ C. Gould, ${ }^{8}$ \\ L. W. Molenkamp, ${ }^{8}$ T. Dietl, ${ }^{9,10}$ and H. Ohno ${ }^{11,12}$ \\ ${ }^{1}$ Institute of Physics, ASCR v.v.i., Cukrovarnická 10, 16253 Praha 6, Czech Republic \\ ${ }^{2}$ School of Physics and Astronomy, University of Nottingham, Nottingham NG7 2RD, United Kingdom \\ ${ }^{3}$ Department of Physics, Texas A\&M University, College Station, Texas 77843-4242, USA \\ ${ }^{4}$ Department of Physics, University of Texas at Austin, Austin, Texas 78712-1081, USA \\ ${ }^{5}$ Institute of Physics, ASCR v.v.i., Na Slovance 2, 18221 Praha 8, Czech Republic \\ ${ }^{6}$ Department of Physics, Korea University, Seoul 136-701, Korea \\ ${ }^{7}$ Hitachi Cambridge Laboratory, Cambridge CB3 OHE, United Kingdom \\ ${ }^{8}$ Physikalisches Institut (EP 3), Universität Würzburg, Am Hubland, 97074 Würzburg, Germany \\ ${ }^{9}$ Institute of Physics, Polish Academy of Science, Aleja Lotnikow 32/46, PL 02-668 Warszawa, Poland \\ ${ }^{10}$ Institute of Theoretical Physics, Warsaw University, PL 00-681 Warszawa, Poland \\ ${ }^{11}$ Laboratory for Nanoelectronics and Spintronics, Research Institute of Electrical Communication, Tohoku University, Katahira 2-1-1, \\ Aoba-ku, Sendai 980-8577, Japan \\ ${ }^{12}$ ERATO Semiconductor Spintronics Project, Japan Science and Technology Agency, Kitamemachi 1-18, Aoba-ku, \\ Sendai 980-0023, Japan
}

(Received 9 July 2007; revised manuscript received 27 August 2007; published 18 September 2007)

\begin{abstract}
We discuss the character of states near the Fermi level in Mn-doped GaAs, as revealed by a survey of dc transport and optical studies over a wide range of Mn concentrations. A thermally activated valence-band contribution to de transport, a midinfrared peak at energy $\hbar \omega \approx 200 \mathrm{meV}$ in the ac conductivity, and the hot photoluminescence spectra indicate the presence of an impurity band in low-doped $(\ll 1 \% \mathrm{Mn})$ insulating GaAs:Mn materials. Consistent with the implications of this picture, both the impurity-band ionization energy inferred from the dc transport and the position of the midinfrared peak move to lower energies, and the peak broadens with increasing Mn concentration. In metallic materials with $>2 \%$ doping, no traces of Mn-related activated contribution can be identified in dc transport, suggesting that the impurity band has merged with the valence band. No discrepancies with this perception are found when analyzing optical measurements in the high-doped GaAs:Mn. A higher-energy $(\hbar \omega \approx 250 \mathrm{meV})$ midinfrared feature which appears in the metallic samples is associated with inter-valence-band transitions. Its redshift with increased doping can be interpreted as a consequence of increased screening, which narrows the localized-state valence-band tails and weakens higher-energy transition amplitudes. Our examination of the dc and ac transport characteristics of GaAs:Mn is accompanied by comparisons with its shallow acceptor counterparts, confirming the disordered valence-band picture of high-doped metallic GaAs:Mn material.
\end{abstract}

DOI: 10.1103/PhysRevB.76.125206

PACS number(s): 75.50.Pp, 71.55.Eq, 75.47.-m

\section{INTRODUCTION}

GaAs is an intermediate band-gap III-V semiconductor, with $E_{g}=1.5 \mathrm{eV}$ at low temperatures, in which an isolated Mn impurity substituting for $\mathrm{Ga}$ functions as an acceptor with an impurity binding energy of intermediate strength, $E_{a}^{0}=0.11 \mathrm{eV} \cdot{ }^{1-5}$ Beyond a critical Mn concentration, Mndoped GaAs exhibits a phase transition to a state in which the Mn impurity levels overlap sufficiently strongly that the ground state is metallic, i.e., that states at the Fermi level are not bound to a single or a group of $\mathrm{Mn}$ atoms but are delocalized across the system. ${ }^{6,7}$ In the metallic regime, Mn can, like a shallow acceptor (e.g., $\mathrm{C}, \mathrm{Be}, \mathrm{Mg}$, and $\mathrm{Zn}$ ), provide delocalized holes with a low-temperature density comparable to Mn density, $N_{\mathrm{Mn}} \cdot{ }^{8-10}$ ( $x=1 \% \mathrm{Mn}$ doping corresponds to $N_{\mathrm{Mn}}=2.2 \times 10^{20} \mathrm{~cm}^{-3}$ in GaAs:Mn.) The transition to the metallic state occurs at larger doping in GaAs when doped with Mn than when doped with shallow acceptors. Experimentally, the transition appears to occur between $N_{\mathrm{Mn}} \approx 1$ $\times 10^{20}$ and $5 \times 10^{20} \mathrm{~cm}^{-3}$, as compared to the $\sim 10^{18} \mathrm{~cm}^{-3}$ critical density for the shallow acceptors in GaAs. ${ }^{11}$

The hole binding potential of an isolated substitutional $\mathrm{Mn}$ impurity is composed of long-range Coulomb, shortranged central cell, and $s p$ - $d$ kinetic-exchange potentials. ${ }^{3}$ Because of the kinetic exchange and central cell interactions, $\mathrm{Mn}$ acceptors are more localized than shallow acceptors. A crude estimate of the critical metal-insulator transition density can be obtained with a short-range potential model, using the experimental binding energy, and assuming an effective mass of valence-band holes, $m^{*}=0.5 m_{e}$. This model implies an isolated acceptor level with effective Bohr radius $a_{0}=\left(\hbar^{2} / 2 m^{*} E_{a}^{0}\right)^{1 / 2}=10 \AA$. The radius $a_{0}$ then equals the $\mathrm{Mn}$ impurity spacing scale $N_{\mathrm{Mn}}^{-1 / 3}$ at $N_{\mathrm{Mn}} \approx 10^{21} \mathrm{~cm}^{-3}$. This explains qualitatively the higher metal-insulator-transition critical density in Mn-doped GaAs compared to the case of systems doped with shallow, more hydrogenic-like acceptors which have binding energies $E_{a}^{0} \approx 30 \mathrm{meV}$.,

The intermediate and magnetic character of extrinsic GaAs:Mn makes the physics near its metal-insulator transition even more complex than in the shallow, nonmagnetic 
acceptor counterparts and difficult to describe quantitatively. However, as it is often the case in semiconductors, important phenomena occur at or near this transition doping region. In GaAs:Mn, the most remarkable among these is the onset of carrier mediated ferromagnetism. The understanding of magnetic properties of these doped semiconductors which are close to the metal-insulator transition can, therefore, only emerge from the understanding of the spectroscopic nature of carriers in this regime.

Unlike the metal-insulator phase transition, which is sharply defined in terms of the temperature $T=0$ limit of the conductivity, the crossover in the character of states near the Fermi level in semiconductors with increased doping is gradual. ${ }^{7,12-15}$ At very weak doping, the Fermi level resides inside a narrow impurity band (assuming some compensation), separated from the valence band by an energy gap of magnitude close to the impurity binding energy. In this regime, strong electronic correlations are an essential element of the physics and a single-particle picture has limited utility. Well into the metallic state, on the other hand, the impurities are sufficiently close together, and the long-range Coulomb potentials which contribute to the binding energy of an isolated impurity are sufficiently screened that the system is best viewed as an imperfect crystal with disorder-broadened and shifted host Bloch bands. In this regime, electronic correlations are usually less strong and a single-particle picture often suffices. (Note that the short-range components of the Mn binding energy in GaAs, which are not screened by the carriers, move the crossover to higher dopings and contribute significantly to carrier scattering in the metallic state.)

Although neither picture is very helpful for describing the physics in the crossover regime, which spans some finite range of dopings, the notion of the impurity band on the lower doping side from the crossover and of the disordered host band on the higher doping side from the crossover still have a clear qualitative meaning. The former implies that there is a deep minimum in the density of states between separate impurity and host band states. In the latter case, the impurity band and the host band merge into one inseparable band whose tail may still contain localized states, depending on the carrier concentration and disorder. We note that terms overlapping and merging impurity and valence bands describe the same basic physics in GaAs:Mn. This is because the $\mathrm{Mn}$-acceptor states span several unit cells even in the very dilute limit, and many unit cells as the impurity band broadens with increasing doping. The localized and delocalized Bloch states then have a similarly mixed As-Ga-Mn spd character. This applies to systems on either side of the metalinsulator transition. We also point out that for randomly distributed Mn dopants in GaAs, the impurity band is not properly modeled as a Bloch-like band with a weak dispersion (heavy effective mass).

The impurity band and the disordered valence-band regimes are schematically illustrated in Fig. 1. In Secs. II-IV, we discuss a survey of previously published dc and ac transport data and new dc measurements of GaAs:Mn materials grown in the Nottingham ${ }^{16}$ and Prague ${ }^{17}$ molecular-beamepitaxy (MBE) systems, spanning a wide range of Mn concentrations. Our comprehensive study complements previous investigations of specific narrower doping regions. It shows

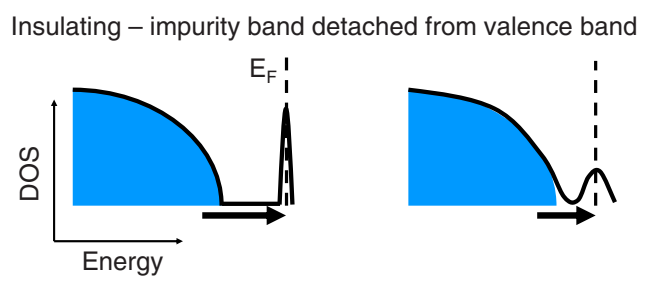

Metallic - merged impurity and valence bands

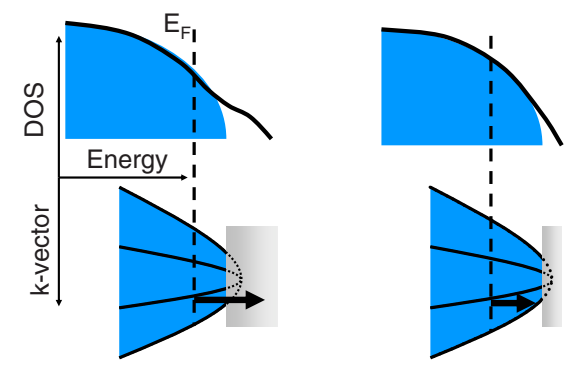

FIG. 1. (Color online) Schematic illustrations of the impurityband regime for low-doped insulating GaAs:Mn (upper panels) and of the disordered-valence-band regime for the high-doped metallic GaAs:Mn (lower panels). Splitting of the bands in the ferromagnetic state is omitted for simplicity. For each regime, the cartoons are arranged from left to right according to increasing doping. Blue areas indicate delocalized states, white and gray areas indicate localized states. Arrows highlight the redshift in the impurity-band ionization energy and the redshift in the inter-valence-band transitions, respectively, which are discussed in Secs. II-IV.

that the picture of the crossover from the impurity band at low dopings to the disordered valence band at high dopings, illustrated in Fig. 1 and generally accepted for the shallow acceptor counterparts to GaAs:Mn, applies well to the intermediate extrinsic GaAs:Mn. The perception of merged impurity and valence bands in highly Mn-doped metallic GaAs is now also firmly established in the microscopic theory community $7,15,18-22$ and provides a qualitative, and often a semiquantitative, description of micromagnetic and magnetotransport characteristics of bulk and microstructured (Ga,Mn)As ferromagnets. ${ }^{6,7,15}$

Our paper, which we believe further establishes the valence-band nature of the Fermi level states in metallic GaAs:Mn, is timely as the topic is still not fully settled. Several recent interpretations of optical measurements in GaAs:Mn (Refs. 23-25) have favored the impurity-band picture of highly doped metallic materials. These works provided additional motivation for the analysis presented here, for which we conclude that the optical data in the high-doped GaAs:Mn are not inconsistent with the disordered valenceband nature of states near the Fermi energy, inferred from dc transport.

Finally, before moving to the next sections, let us remark that comparisons between samples with different nominal Mn concentrations have to be treated with caution. The Mn doping parameter should only be considered as an approximate guideline for qualitative discussions of doping trends. This applies, namely, to epitaxial materials with higher $\mathrm{Mn}$ concentrations in which nominal, growth-rate-controlled Mn 

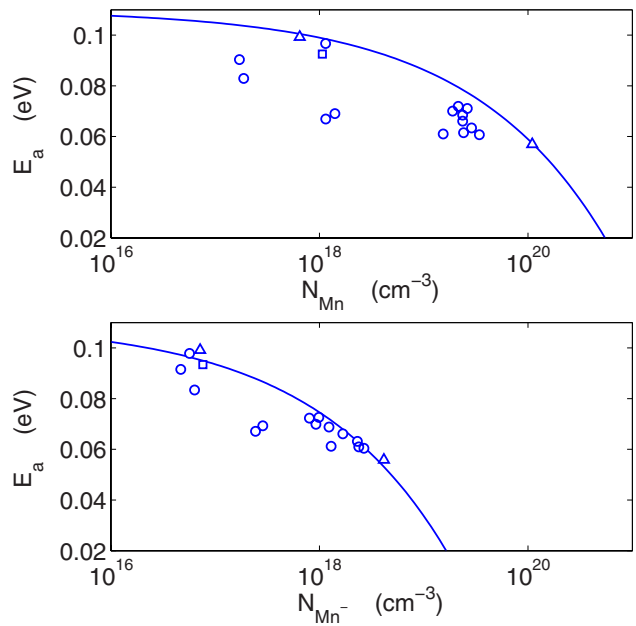

FIG. 2. (Color online) Mn doping dependent energy gap between the impurity band and valence band, measured in the bulk equilibrium-grown GaAs:Mn and plotted as a function of total Mn density (top panel) and ionized Mn denisty (bottom panel). All data are replotted from Ref. 2 .

doping parameter can deviate significantly from the amount of $\mathrm{Mn}$ impurities incorporated in the acceptorlike Gasubstitutional positions. Moreover, the partial densities of various types of $\mathrm{Mn}$ impurities and of other compensating and structural defects can vary significantly depending on applied growth conditions.

\section{LOW-DOPED INSULATING GaAs:Mn SYSTEMS WITH IMPURITY BANDS}

Narrow impurity bands are expected and have been clearly observed in Mn-doped GaAs samples with carrier densities much lower than the metal-insulator-transition density, for example, in equilibrium-grown bulk materials with $N_{\mathrm{Mn}}=10^{17}-10^{19} \mathrm{~cm}^{-3}, 2,26,27$ The energy gap between the impurity band and the valence band, $E_{a}$, can be measured by studying the temperature dependence of longitudinal and Hall conductivities, which show an activated behavior because of thermal excitation of holes from the impurity band to the much more conductive valence band. ${ }^{2,27,28}$ Examples of these measurements are shown in Figs. 2-4.

The activation energy decreases with increasing Mn density, following roughly the form ${ }^{2}$

$$
E_{a}=E_{a}^{0}\left[1-\left(N_{\mathrm{Mn}} / N_{\mathrm{Mn}}^{c}\right)^{1 / 3}\right] .
$$

The lowering of impurity binding energies at larger $N_{\text {Mn }}$, which is expected to scale with the mean impurity separation as expressed in Eq. (1), is apparent already in the equilibrium-grown bulk materials with $N_{\mathrm{Mn}}$ $=10^{17}-10^{19} \mathrm{~cm}^{-3}$. The trend continues in epitaxially grown (at $400{ }^{\circ} \mathrm{C}$ ) GaAs:Mn with $N_{\mathrm{Mn}} \approx 1 \times 10^{19}$ and 6 $\times 10^{19} \mathrm{~cm}^{-3} .{ }^{29}$ When this trend is extrapolated using Eq. (1), it places the disappearance of the gap at Mn dopings of $\sim 1 \%$. Note that this estimate has a large scatter depending also on whether all Mn or only ionized Mn impurities are considered when fitting the data by Eq. (1) (see Fig. 2). ${ }^{2}$

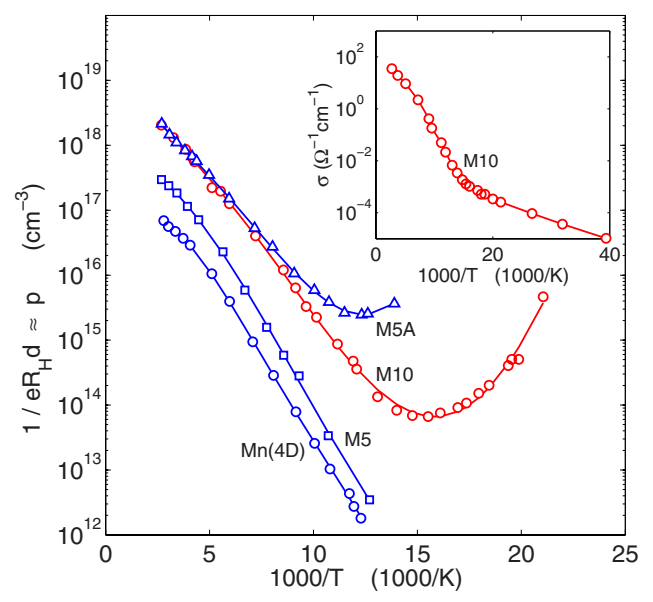

FIG. 3. (Color online) Hole densities determined from the Hall coefficient measurement as a function of temperature in bulk equilibrium-grown GaAs:Mn. Inset: Temperature-dependent longitudinal conductivity (from Refs. 2 and 26). Sample $\mathrm{Mn}$ (4D) has Mn density of $1.6 \times 10^{17} \mathrm{~cm}^{-3} ;$ M5, $1.1 \times 10^{18} \mathrm{~cm}^{-3} ;$ M10, 1.9 $\times 10^{19} \mathrm{~cm}^{-3}$; and Mn5A, $9.3 \times 10^{19} \mathrm{~cm}^{-3}$.

For $N_{\mathrm{Mn}} \approx 1 \times 10^{19} \mathrm{~cm}^{-3}(x \approx 0.06 \%), E_{a} \approx 70 \mathrm{meV}$ and a sharp crossover from impurity-band hopping conduction to activated valence-band conduction is observed at $T$ $\approx 80-100 \mathrm{~K}$ (see Figs. 3 and 4). Near the crossover, $N_{\mathrm{Mn}} / p \sim 10^{3}-10^{5}$, where $p$ is the valence-band hole density, consistent with the much higher mobility of valence-band holes compared to impurity-band holes in this regime for which the distinction is clearly still valid and useful. (Note that in the bulk equilibrium-grown samples, the temperature at which the crossover occurs appears to be very sensitive to the detailed disorder configuration at this Mn doping level, with some samples being strongly insulating and others showing signatures of filamentary conducting channels. $\left.{ }^{26,27,29}\right)$ For the $N_{\mathrm{Mn}} \approx 6 \times 10^{19} \mathrm{~cm}^{-3}(x \approx 0.3 \%)$ epitaxial sample,$^{29}$ the crossover from impurity-band conduction to activated valence-band conduction can still be identified at $T \approx 140 \mathrm{~K}$ (see Fig. 4) and $E_{a} \approx 50 \mathrm{meV}$.

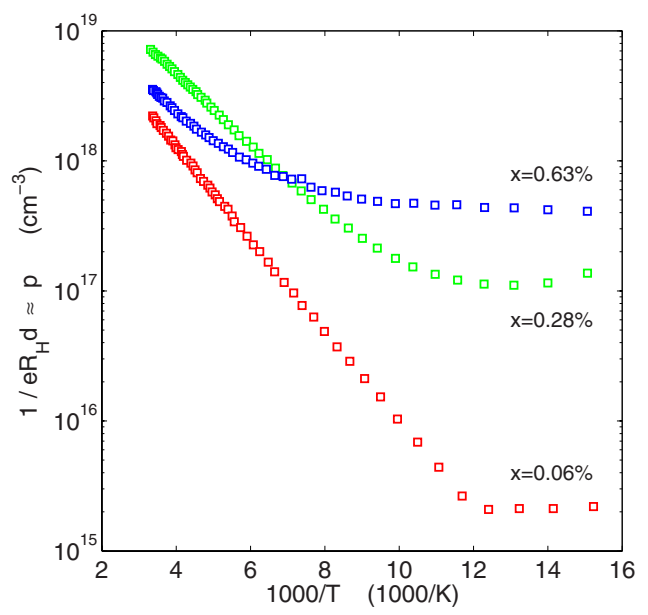

FIG. 4. (Color online) Same as Fig. 3 for MBE grown (at $400{ }^{\circ} \mathrm{C}$ ) GaAs:Mn (from Ref. 29). 


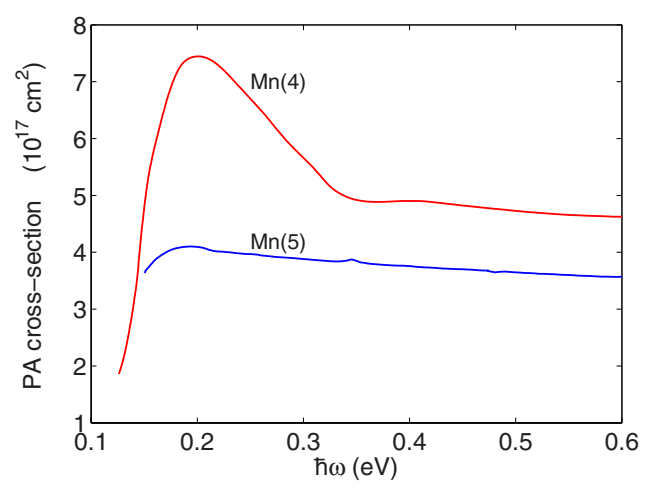

FIG. 5. (Color online) Infrared photoabsorption cross section measurements in bulk GaAs:Mn (from Ref. 26). $M n(4), 1.7$ $\times 10^{17} \mathrm{~cm}^{-3} ; \operatorname{Mn}(5), 9.3 \times 10^{18} \mathrm{~cm}^{-3}$.

In the dilute limit where the system has a narrow insulating impurity band, the ac conductivity is expected to show a broad midinfrared feature due to impurity band to valence band transitions, peaked at energy between $E_{a}$ and $2 E_{a} \cdot{ }^{26,30,31}$ Lower energy peak positions correspond to shallow acceptors with weakly bound impurity states composed of a narrow range of valence-band wave vector Fourier components. The peak near $2 E_{a}$ and a line shape $\sigma(\hbar \omega) \sim E_{a}^{1 / 2}(\bar{\omega}$ $\left.-E_{a}\right)^{3 / 2} /(\hbar \omega)^{3}$ correspond to a short-range impurity potential, for which a larger interval of valence-band states contributes to the transition amplitude. ${ }^{26,30,31}$ Consistent with these expectations, a peak at $\hbar \omega \approx 200 \mathrm{meV}$ is observed in the weakly doped $\left(N_{\mathrm{Mn}} \sim 10^{17} \mathrm{~cm}^{-3}\right)$ GaAs:Mn samples (see Fig. 5). ${ }^{1,26,32}$ At $N_{\mathrm{Mn}} \sim 10^{19} \mathrm{~cm}^{-3}$, the peak is slightly redshifted $(\hbar \omega \approx 180 \mathrm{meV})$ and significantly broadened. ${ }^{26}$ Both the redshifting and the broadening are expected consequences of increasing overlap between acceptor levels.

In the dilute doping disorder free limit, hot photoluminescence (HPL) can provide another optical spectroscopy tool, when combined with a reliable measure of the heavy-hole kinetic energy at the valence band to conduction band excitation energy, to measure the position of a narrow impurity band with respect to the top of the valence band. ${ }^{33}$ For a GaAs:Mn sample doped with $\sim 10^{17} \mathrm{~cm}^{-3}$ of $\mathrm{Mn}$, a HPL spectrum has been obtained ${ }^{24}$ (see also Fig. 10) that is consistent with excitations from the valence band to the conduction band, followed by transitions of the hot electrons (before their energy is dissipated in the conduction band) into the impurity level. An edge can be identified in the spectrum which corresponds to an impurity level $\sim 120 \pm 10 \mathrm{meV}$ above the top of the valence band, in agreement with the above dc and infrared ac transport data and other experiments. ${ }^{1-5}$

We conclude that in the low doping regime, the temperature-dependent dc transport data and the ac conductivity data consistently indicate the presence of an impurity band and the decrease in $E_{a}$ as doping brings the impurity band closer to the valence band. Note that the activated dc transport marker of the impurity band is common to both the intermediate $\mathrm{Mn}$ acceptor and the shallow acceptors in GaAs. ${ }^{11}$ The infrared ac conductivity feature, on the other hand, appears to be a more subtle and less reliable tool for identifying the presence of the impurity band. To our knowledge, there are no reports of the impurity-band transitions in infrared ac measurements for GaAs doped with shallow acceptors. In these systems, even when weakly doped and still insulating, the reported infrared ac conductivity peaks are ascribed to inter-valence-band transitions due to nonzero thermally activated or metallic hole densities in the valence band. ${ }^{34-36}$ The inter-valence-band transitions in metallic GaAs:Mn will be analyzed in detail in Sec. IV.

\section{IMPURITY-BAND TO DISORDERED-VALENCE-BAND CROSSOVER AND HIGH-DOPED METALLIC GaAs:Mn}

At higher $\mathrm{Mn}$ doping around $\approx 1 \%$, the conductivity curves can no longer be separated into an impurity-band dominated contribution at low temperatures and an activated valence-band dominated contribution at higher temperatures, even though the samples are still insulators (see Fig. 6). In metallic materials with $x>2 \%$, no signatures of Mn-related hole activation is observed when temperature is swept to values as high as $500 \mathrm{~K}$, i.e., to $T \approx E_{a}^{0} / 2$, and before the intrinsic conductance due to activation across the GaAs band gap takes over (see Fig. 6).

For completeness, we show in Fig. 7 temperaturedependent resistivity curves for a set of metallic, annealed high conductivity ferromagnetic samples, which, within experimental uncertainty of $20 \%$, show no compensation ${ }^{10}$ and conductivities up to $\approx 900 \Omega^{-1} \mathrm{~cm}^{-1}$. We note here that the high-doped metallic GaAs:Mn samples show only weak temperature dependence of the conductivity associated with the onset of ferromagnetism and that the conductivity varies slowly with Mn composition in the metallic samples but changes dramatically in going from $1 \%$ to $1.5 \% \mathrm{Mn}$ in these samples. We also note that no marked dependence of the hole density on temperature is observed for metallic samples. ${ }^{8}$

The absence of the impurity band in high-doped GaAs:Mn is further evident by comparisons with other related materials (see Fig. 8) for which the disordered-valenceband picture has not been questioned. The narrower-gap InAs:Mn counterparts to GaAs:Mn, e.g., in which Mn acts as a shallower acceptor, have similarly low magnitudes and similar temperature dependence of the conductivity. ${ }^{37-39}$ Comparably low conductivities (or mobilities) are also found in epitaxially grown GaAs doped with $\sim 10^{20} \mathrm{~cm}^{-3}$ of the shallow nonmagnetic acceptor $\mathrm{Mg} .{ }^{40}$ Note that the highest mobilities in $p$-type GaAs with the doping levels $\sim 10^{20}-10^{21} \mathrm{~cm}^{-3}$ are achieved with $\mathrm{Zn}$ and $\mathrm{C}$, and these are only five to ten times larger as compared to GaAs:Mn (see Fig. 8). ${ }^{41,42}$

We also remark that valence-band calculations treating disorder in the Born approximation overestimate the experimental dc conductivities of metallic GaAs:Mn by less than a factor of $10,{ }^{43,44}$ and that any sizable discrepancy is removed by exact-diagonalization calculations ${ }^{45}$ (see also Fig. 13), which account for strong disorder and localization effects. This provides another confirmation for the plausibility of the disordered valence-band picture.

In Fig. 9, we illustrate the dependence of the metallicity of the experimental GaAs:Mn films on doping by plotting 

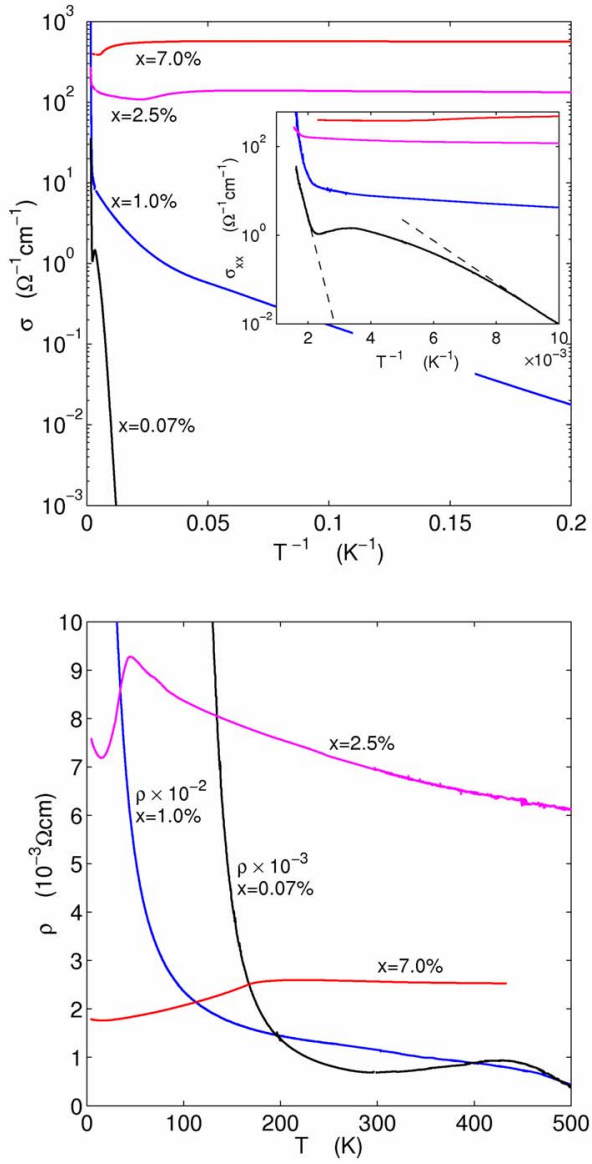

FIG. 6. (Color online) Comparison of longitudinal conductivities (upper panel) and resistivities (lower panel) of $0.07 \%$ and $1 \%$ doped insulating paramagnetic GaAs:Mn, and $2.5 \%$ and $7 \%$ Mndoped ferromagnetic ( $T_{c}$ approximately 40 and $170 \mathrm{~K}$, respectively) metallic GaAs:Mn grown in the Prague MBE system with $\mathrm{As}_{4}$ flux. The $0.07 \%$ doped material was grown at $530 \mathrm{~K}$, the other materials were grown by low-temperature MBE (240-200 K). The $0.07 \%$ doped sample shows clear GaAs:Mn impurity band-valence band activation at low temperatures, impurity-band exhaustion, and the onset of activated transport over GaAs band gap at high temperatures. The $2.5 \%$ and $7 \%$ doped samples show only weak conductance variations associated with the onset of ferromagnetism until the activated transport over GaAs band gap takes over.

estimated experimental values for the dimensionless parameter $k_{F} \Lambda=\hbar \mu k_{F}^{2} / e$. Here, $k_{F}=\left(6 \pi^{2} p / D\right)^{1 / 3}$ is the Fermi wave vector obtained from the experimental hole densities assuming a fully polarized $(D=1)$ or unpolarized $(D=2)$ parabolic band, and $\Lambda$ is the mean free path obtained from experimental mobilities $\mu$ and assuming the Drude conductivity formula. In the annealed samples with low concentration of compensating defects, $k_{F} \Lambda$ systematically increases with Mn doping, while for the as-grown metallic GaAs:Mn materials, $k_{F} \Lambda$ is nearly constant due to compensation by, primarily, interstitial Mn donors. ${ }^{10}$ As expected for systems not far from the metal-insulator transition, these estimates of the $k_{F} \Lambda$ parameter indicate that even the most conductive experimental GaAs:Mn materials are still "dirty" metals with $k_{F} \Lambda<5$. We also note that the above estimates yield $\Lambda$
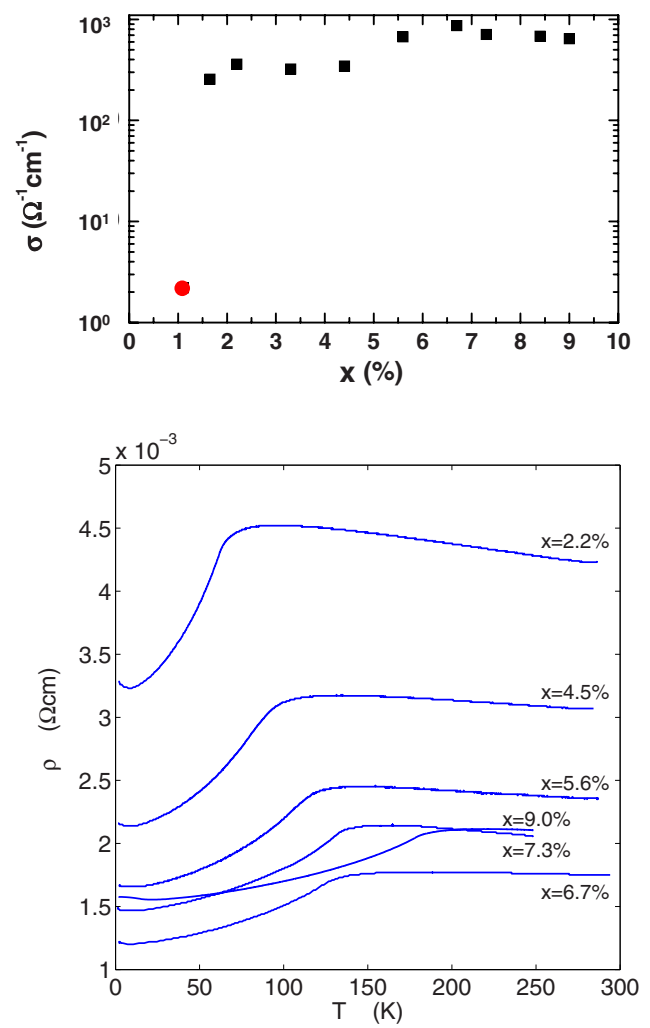

FIG. 7. (Color online) de transport measurements in optimally annealed, metallic ferromagnetic GaAs:Mn $\left(T_{c}\right.$ 's between approximately 50 and $170 \mathrm{~K}$ ) grown by the Nottingham low-temperature MBE system with $\mathrm{As}_{2}$ flux. Upper panel: Conductivities at $10 \mathrm{~K}$ plotted as a function of $\mathrm{Mn}$ doping; insulating 1\% doped material grown under the same conditions is included for comparison (red dot). Lower panel: Temperature dependence of longitudinal resistivities.

$\sim 1 \mathrm{~nm}$, which is consistent with the typical separation of the Mn impurities in the high-doped metallic materials.

Similarities between GaAs doped with Mn and with shallow acceptors, $\mathrm{Zn}$ in particular, are also found in the experimental HPL spectra, as shown in Fig. $10 .^{24,33}$ While for doping levels $\sim 10^{17} \mathrm{~cm}^{-3}$ a sharp onset of the luminescence at frequencies corresponding to transitions into the impurity bands in these materials is clearly detected, the HPL impurity-band marker is not apparent at high dopings. For the metallic GaAs:Zn with $N_{\mathrm{Zn}} \approx 10^{19} \mathrm{~cm}^{-3}$ and for the $4 \%$ doped GaAs:Mn, the spectra show only broad luminescence with no clear indication of an onset but, rather, a featureless decrease continuing out toward the laser energy.

The first infrared ac conductivity measurements in highdoped GaAs:Mn, reported in Refs. 48 and 49, were inconclusive. Only two samples were compared in both of these works, with approximately 3\% and 5\% nominal Mn doping. The absence of a clear midinfared peak in the $5 \%$ material in Ref. 48 and the reentrant insulating behavior of the higher doped sample in Ref. 49 suggest that the growth at this early stage had not been optimized so as to minimize the formation of unintentional defects and to achieve appreciable growth reproducibility.

The more recent and more systematic infrared absorption measurements ${ }^{23,32,50}$ for an insulating sample close to the 


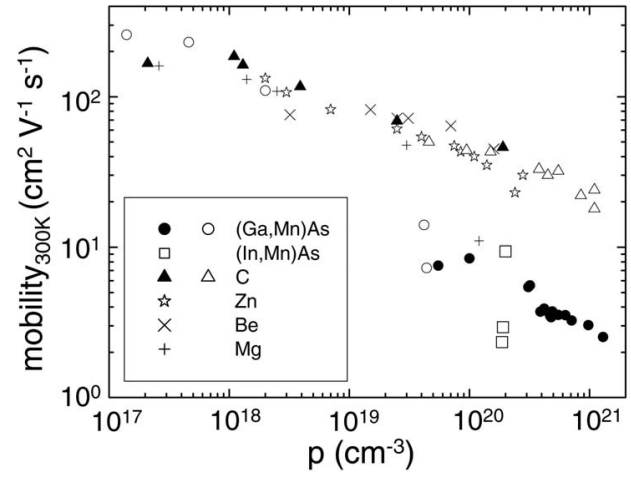

FIG. 8. (Color online) Room temperature Hall mobilities as a function of hole concentration for GaAs doped with Mn (filled circles, present results; open circles, Ref. 46), C (filled triangles, present results; open triangles, Ref. 42), Zn (stars, Ref. 41), Be (X, Ref. 47), and Mg (plus, Ref. 40), as well as InAs doped with Mn (square, Refs. ${ }^{37,38}$ ). Hole concentrations were obtained from lowtemperature high-field Hall measurements for the ferromagnetic metallic GaAs:Mn and InAs:Mn films, and from room temperature Hall measurements for the other films.

metal-insulator transition and for a set of metallic highly doped GaAs:Mn materials are shown in Fig. 11. The ac conductivity of the $1.7 \%$ doped insulating sample is featureless
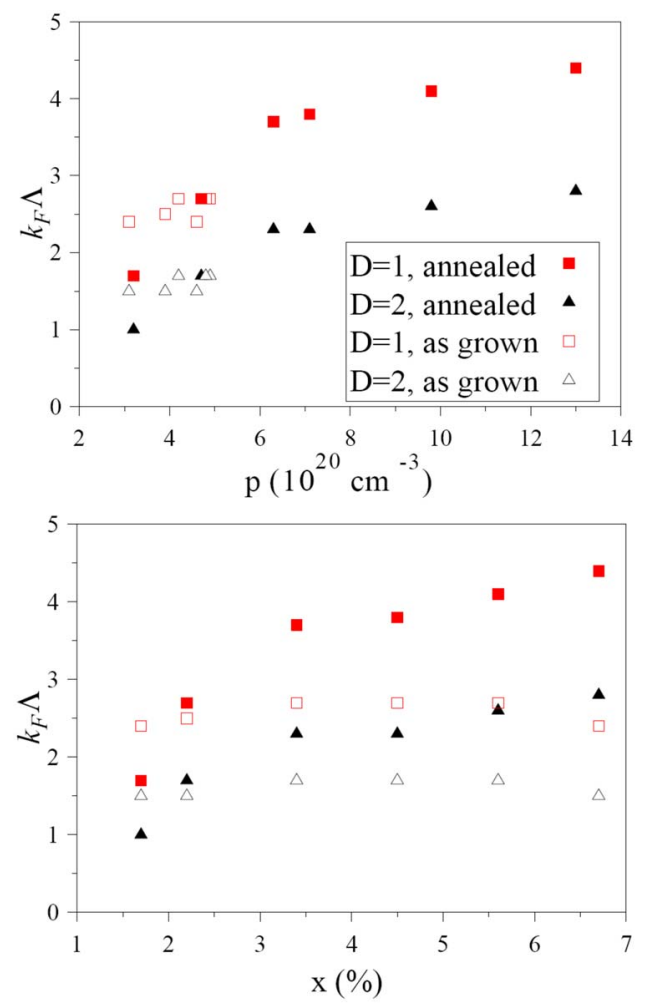

FIG. 9. (Color online) Estimates of the $k_{F} \Lambda$ parameter as a function of hole density (upper panel) and Mn doping (lower panel) for metallic GaAs:Mn epilayers obtained from the measured mobilities and hole densities, and assuming parabolic fully polarized $(D=1)$ or unpolarized $(D=2)$ heavy-hole valence bands and the Drude form of dc conductivity.
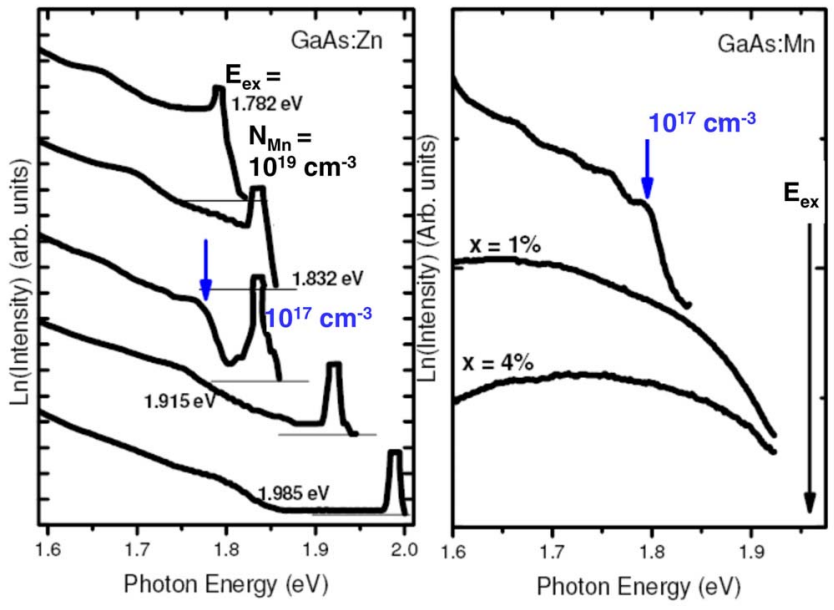

FIG. 10. (Color online) Left panel: HPL spectra of GaAs doped with $\sim 10^{17} \mathrm{~cm}^{-3}$ of $\mathrm{Zn}$ (see blue arrow) and $\sim 10^{19} \mathrm{~cm}^{-3}$ of $\mathrm{Zn}$ (from Ref. 33, replotted in logarithmic scale). Right panel: HPL spectra of GaAs doped with $\sim 10^{17} \mathrm{~cm}^{-3}$ of $\mathrm{Mn}$ (see blue arrow), and of $\approx 1 \%$ and $\approx 4 \%$ GaAs:Mn materials (from Ref. 24). $E_{e x}$ is the HPL excitation energy. Arrows indicate the spectral feature corresponding to impurity-band transitions in the low-doped materials.

in the midinfrared region..$^{32,50}$ This can be interpreted as a natural continuation of the trend, started in the low doped samples, in which the impurity band moves closer to the valence band and broadens with increasing doping, becoming eventually undetectable in the ac conductivity spectra. Note that the disappearance of the impurity-band transitions can, in principle, result also from a strong compensation..$^{50} \mathrm{~A}$ strong unintentional compensation in epitaxial GaAs:Mn at $\sim 1 \%$ doping level is not typical, however. The $1.7 \%$ doped sample from Ref. 50 has a room temperature conductivity in the dc limit of about $5 \Omega^{-1} \mathrm{~cm}^{-1}$, which presumably drops to a much lower value at $5 \mathrm{~K} .{ }^{50}$ This is consistent with the $\sigma(T)$ dependence of the insulating $1 \%$ sample from the series of epitaxial materials in Fig. 6 which show no signatures of strong compensation over the entire range of doping from $0.07 \%$ to $7 \%$.

A new midinfrared feature emerges in the metallic $>2 \%$ doped samples at frequencies $\hbar \omega \approx 250 \mathrm{meV}$, and is then redshifted by $\approx 80 \mathrm{meV}$ as the doping is further increased to approximately $7 \%$ without showing a marked broadening (see Fig. 11).23,32 The association of this peak to an impurity band is implausible because of (i) the absence of the activated dc-transport counterpart in the high-doped metallic samples, (ii) the blueshift of this midinfrared feature with respect to the impurity-band transition peak in the $N_{\mathrm{Mn}}$ $\sim 10^{19} \mathrm{~cm}^{-3}$ sample, (iii) the appearance of the peak at frequency above $2 E_{a}^{0}$, and (iv) the absence of a marked broadening of the peak with increased doping. In the following section, we argue that this peak in the infrared conductivity of metallic GaAs:Mn is consistent with the picture of merged impurity and valence bands in these materials.

\section{REDSHIFT OF THE MIDINFRARED ABSORPTION ABOVE INSULATOR-TO-METAL TRANSITION}

A midinfrared peak has been observed in metallic GaAs heavily doped $\left(\sim 10^{19}-10^{20} \mathrm{~cm}^{-3}\right)$ with shallow hydrogenic 

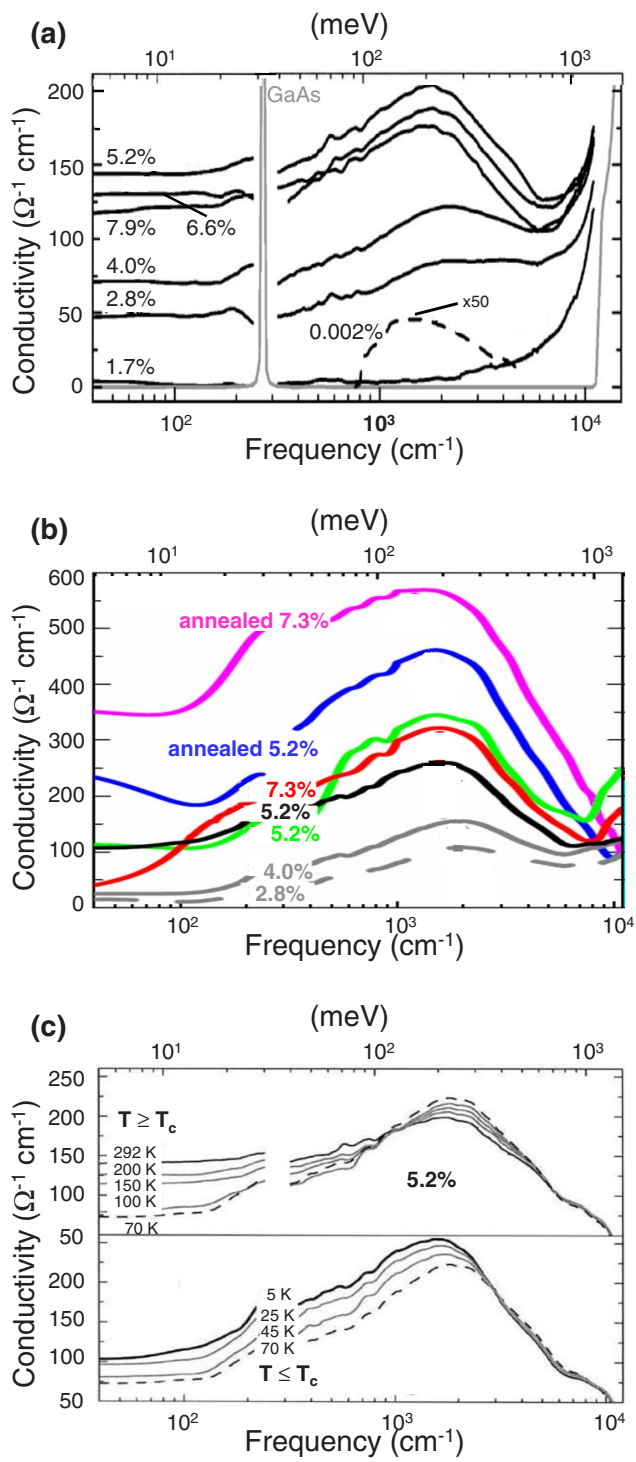

FIG. 11. (Color online) Infrared absorption measurements of GaAs:Mn. Top panel: Comparison of GaAs, low-doped GaAs:Mn, and high-doped as-grown GaAs:Mn materials at $292 \mathrm{~K}$. Middle panel: Comparison of high-doped as-grown and annealed GaAs:Mn samples at $7 \mathrm{~K}$. Bottom panel: Absorption measurements for the 5.2\% as-grown GaAs:Mn below and above the ferromagnetic transition temperature. Data taken from Refs. 23, 32, and 50.

carbon acceptors and explained in terms of inter-valenceband transitions between heavy holes and light holes. ${ }^{36}$ This peak blueshifts with increasing doping as the Fermi energy moves further into the valence and, consequently, the transitions move to higher energies (see Fig. 12). ${ }^{36,44}$ The conclusions of previous sections suggest that the midinfrared peaks in the metallic GaAs:Mn materials should be of a qualitatively similar origin. The redshift seen in GaAs:Mn can be understood by recalling that while GaAs doped with $\sim 10^{19}-10^{20} \mathrm{~cm}^{-3} \mathrm{C}$ is already far on the metallic side, GaAs doped with $\sim 10^{20}-10^{21} \mathrm{~cm}^{-3}$ of $\mathrm{Mn}$ is still close to the metal-insulator transition.

The merging of the impurity band and the valence band near the transition is illustrated schematically in Fig. 1. For

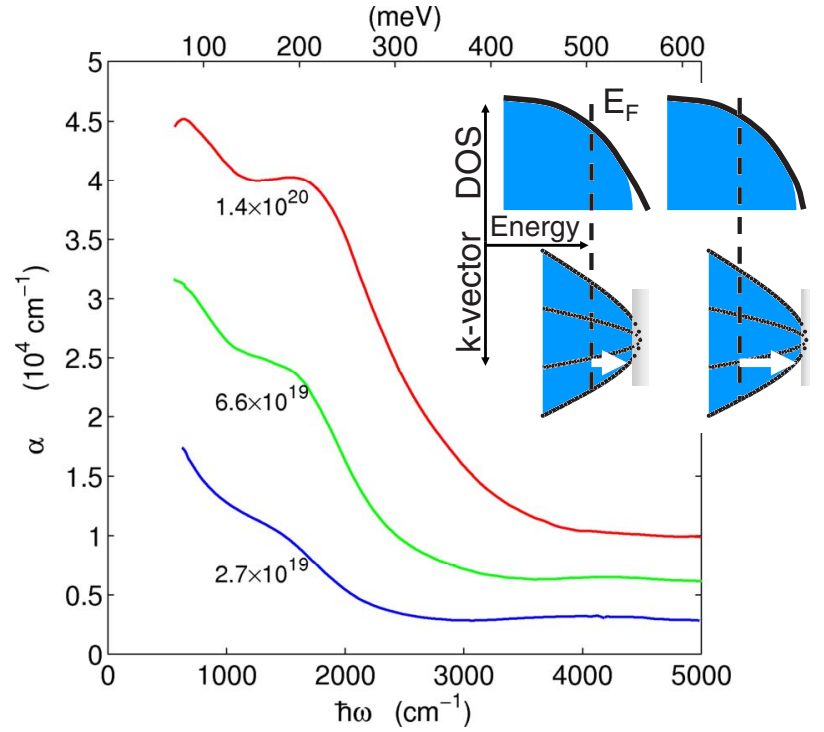

FIG. 12. (Color online) Infrared absorption measurements of metallic GaAs:C (from Ref. 36). The inset illustrates the origin of the blueshift of the midinfrared peak in these highly metallic systems.

metallic GaAs:Mn close to the metal-insulator transition, states in the band tail can still be expected to remain localized and spread out in energy (see lower panels in Fig. 1). The redshift of the inter-valence-band transitions can result from an increased metallicity with increasing doping. At lower doping, the abundance of localized states in the broad valence-band tail enables transitions which take spectral weight from the low-frequency region and provide a channel for higher-energy transitions. As doping increases, the valence-band tail narrows because of increased screening. The inter-valence-band absorption peak then redshifts as the low-frequency part of the spectrum adds spectral weight.

A detailed quantitative theoretical modeling of the infrared conductivity, or any other transport or micromagnetic characteristics, of high-doped GaAs:Mn is inherently difficult due to the strong disorder and electron correlation effects near the metal-insulator transition and due to the lack of accurate characterization of the doping parameters and unintentional disorder in the experimental materials. Nevertheless, a qualitative illustration of the complex behavior of the midinfrared peak in strongly disordered valence bands can be found in finite-size exact-diagonalization calculations reported in Ref. 45, which were intended to address the influence of strong disorder on the ac conductivity in metallic GaAs:Mn close to the metal-insulator transition. In these numerical data, shown in Fig. 13, the midinfrared feature is shifted to lower energies in the higher hole density more conductive system. In both experimental and numerical calculation cases, the redshift of the peaks is accompanied by an increase of the dc conductivity. In experiment, this correlation is observed both by comparing different samples and, in a given sample, over the entire range of temperatures that was studied, below and above the ferromagnetic transition temperature (see Fig. 11). ${ }^{23,32,50}$

As the metal-insulator transition is approached from the metal side in a multiband system, it appears that the spectral 


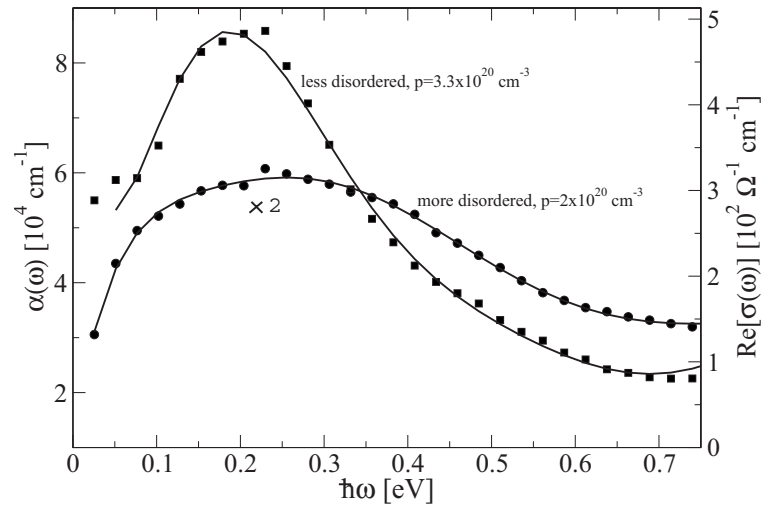

FIG. 13. Exact diagonalization study showing the redshift of the valence-band midinfrared peak for the Kohn-Luttinger model, assuming lower hole concentration and stronger disorder due to larger number of compensating defects (dots) and higher hole density and weaker disorder (rectangles). Lines are tenth-order polynomial fits to the numerical data to guide the eye.

weight that is lost from the disappearing Drude peak, centered on zero frequency, is shifted to much higher energies, perhaps due to transitions to localized valence-band tail states as suggested by the cartoon in Fig. 1. There is a useful sum rule for the conductivity integrated over the energy range corresponding to the semiconductor band gap, provided that the Fermi energy is not too deep in the valenceband and that the valence-band tails do not slide too deeply into the gap. ${ }^{44,45}$ The sum-rule optical masses derived on the basis of the ac conductivity calculations for GaAs:Mn with the Fermi level in the valence band are consistent with experiment to within a factor of $2-3 .^{23,44,45}$ (A more quantitative comparison is hindered by inaccuracies in the theoretical modeling of the GaAs:Mn valence bands and uncertainties associated with the high-frequency cutoff imposed on the experimental sum rules and with the determination of material parameters, hole densities, in particular.)

We emphasize that no general practical sum rule exists for the low-frequency part of the ac conductivity and that a Drude fit of the spectra near the dc limit ${ }^{23}$ would tend to strongly overestimate band effective masses as the metalinsulator transition is approached. The Drude fit is invalidated by frequency-dependent quantum corrections near the metal-insulator transition and by the presence of lowfrequency interband transitions. In GaAs:Mn, in particular, most of the experimental ac conductivity curves in Fig. 11 as well as the numerical simulations in Fig. 13 do not show a maximum at zero frequency, ${ }^{23,32,45,50}$ which makes the Drude fitting inapplicable.

Finally, we point out that the absolute conductance values of the midinfrared conductivity peak found experimentally in metallic GaAs:Mn materials, which are expected to be relatively insensitive to disorder, agree with the values predicted theoretically by the inter-valence-band transition calculations. ${ }^{44,45}$ This includes both the calculations treating disorder within the Born approximation, ${ }^{44}$ which overestimate the dc conductivity, and the exact-diagonalization studies shown in Fig. 13, which account for strong disorder and localization effects. ${ }^{45}$

\section{SUMMARY}

In summary, a number of dc transport and optical measurements have been performed during the past four decades to elucidate the nature of states near the Fermi level in GaAs:Mn in the insulating and metallic regimes. A detailed examination of these studies, complemented with additional dc transport data and comparisons with shallow acceptor counterparts to GaAs:Mn, shows that impurity-band markers are consistently seen in the insulating low-doped materials. Similar consistency is found when analyzing experiments in the high-doped metallic materials, in this case, strongly favoring the disordered valence-band picture. Our conclusions support the established theoretical description of GaAs:Mn based on a variety of different $a b$ initio and phenomenological approaches which explain many experimental magnetic and magnetotransport properties of these dilute moment ferromagnetic semiconductors.

\section{ACKNOWLEDGMENTS}

We acknowledge fruitful and stimulating discussions with Dimitri Basov, Kenneth Burch, Elbio Dagotto, Alexander Finkelstein, Konstantin Kikoin, Yuri Kusrayev, Adriana Moreo, Victor Sapega, and Carsten Timm. This work was supported by ONR under Grant No. ONR-N000140610122, by the NSF under Grant No. DMR-0547875, by the SWANNRI, by the SRC-NRI (SWAN), by EU Grant No. NANOSPIN IST-015728, by EPSRC Grant No. GR/S81407/01, by GACR and AVCR Grants Nos. 202/05/0575, 202/04/ 1519, FON/06/E001, FON/06/E002, KAN400100625, AV0Z1010052, and LC510. J. S. is a Cottrell Scholar of Research Corporation. S.-R.Y. acknowledges support from the Second Brain 21 Project.
${ }^{1}$ R. A. Chapman and W. G. Hutchinson, Phys. Rev. Lett. 18, 443 (1967).

${ }^{2}$ J. S. Blakemore, W. J. Brown, M. L. Stass, and D. A. Woodbury, J. Appl. Phys. 44, 3352 (1973).

${ }^{3}$ A. K. Bhattacharjee and C. B. à la Guillaume, Solid State Commun. 113, 17 (2000).

${ }^{4}$ A. M. Yakunin, A. Y. Silov, P. M. Koenraad, J. H. Wolter, W. Van Roy, J. De Boeck, J. M. Tang, and M. E. Flatté, Phys. Rev. Lett.
92, 216806 (2004).

${ }^{5}$ O. Madelung, U. Rössler, and M. Schulz, Impurities and Defects in Group IV Elements, IV-IV and III-V Compounds, LandoltBörnstein, New Series, Group III, Vol. 41A2b, Pt. B (SpringerVerlag, Berlin, 2003).

${ }^{6}$ F. Matsukura, H. Ohno, and T. Dietl, in Handbook of Magnetic Materials, edited by K. H. J. Buschow (Elsevier, Amsterdam, 2002), Vol. 14, p. 1. 
${ }^{7}$ T. Jungwirth, J. Sinova, J. Mašek, J. Kučera, and A. H. MacDonald, Rev. Mod. Phys. 78, 809 (2006).

${ }^{8}$ D. Ruzmetov, J. Scherschligt, D. V. Baxter, T. Wojtowicz, X. Liu, Y. Sasaki, J. K. Furdyna, K. M. Yu, and W. Walukiewicz, Phys. Rev. B 69, 155207 (2004).

${ }^{9}$ A. H. MacDonald, P. Schiffer, and N. Samarth, Nat. Mater. 4, 195 (2005)

${ }^{10}$ T. Jungwirth et al., Phys. Rev. B 72, 165204 (2005).

${ }^{11}$ A. Ferreira da Silva, I. Pepe, B. E. Sernelius, C. Persson, R. Ahuja, J. P. de Souza, Y. Suzuki, and Y. Yang, J. Appl. Phys. 95, 2532 (2004).

${ }^{12}$ B. I. Shklovskii and A. L. Efros, Electronic Properties of Doped Semiconductors (Spinger-Verlag, New York, 1984).

${ }^{13}$ P. A. Lee and T. V. Ramakrishnan, Rev. Mod. Phys. 57, 287 (1985).

${ }^{14}$ M. A. Paalanen and R. N. Bhatt, Physica B 169, 223 (1991).

${ }^{15}$ T. Dietl, J. Phys.: Condens. Matter 19, 165204 (2007).

${ }^{16}$ R. P. Campion, K. W. Edmonds, L. X. Zhao, K. Y. Wang, C. T. Foxon, B. L. Gallagher, and C. R. Staddon, J. Cryst. Growth 247, 42 (2003).

${ }^{17}$ M. Kopecký, J. Kub, E. Busetto, A. Lausi, M. Cukr, V. Novák, K. Olejník, J. P. Wright, and J. Fábry, J. Appl. Crystallogr. 39, 735 (2006).

${ }^{18}$ P. M. Krstajić, F. M. Peeters, V. A. Ivanov, V. Fleurov, and K. Kikoin, Phys. Rev. B 70, 195215 (2004).

${ }^{19}$ E. H. Hwang and S. Das Sarma, Phys. Rev. B 72, 035210 (2005).

${ }^{20}$ M. Wierzbowska, D. Sanchez-Portal, and S. Sanvito, Phys. Rev. B 70, 235209 (2004).

${ }^{21}$ Y. Yildirim, G. Alvarez, A. Moreo, and E. Dagotto (2006), arXiv:cond-mat/0612002 (unpublished).

${ }^{22}$ F. Popescu, C. Sen, E. Dagotto, and A. Moreo (2007), arXiv:0705.0309 (unpublished).

${ }^{23}$ K. S. Burch, D. B. Shrekenhamer, E. J. Singley, J. Stephens, B. L. Sheu, R. K. Kawakami, P. Schiffer, N. Samarth, D. D. Awschalom, and D. N. Basov, Phys. Rev. Lett. 97, 087208 (2006).

${ }^{24}$ V. F. Sapega, M. Moreno, M. Ramsteiner, L. Däweritz, and K. H. Ploog, Phys. Rev. Lett. 94, 137401 (2005).

${ }^{25}$ V. F. Sapega, M. Ramsteiner, O. Brandt, L. Däweritz, and K. H. Ploog, Phys. Rev. B 73, 235208 (2006).

${ }^{26}$ W. J. Brown and J. S. Blakemore, J. Appl. Phys. 43, 2242 (1972).

${ }^{27}$ D. A. Woodbury and J. S. Blakemore, Phys. Rev. B 8, 3803 (1973).
${ }^{28}$ M. P. Marder, Condensed Matter Physics (Wiley, New York, 2000), supplementary material.

${ }^{29}$ M. Poggio, R. C. Myers, N. P. Stern, A. C. Gossard, and D. D. Awschalom, Phys. Rev. B 72, 235313 (2005).

${ }^{30}$ W. W. Anderson, Solid-State Electron. 18, 235 (1975).

${ }^{31}$ V. N. Fleurov and K. A. Kikoin, J. Phys. C 15, 3523 (1982).

${ }^{32}$ E. J. Singley, R. Kawakami, D. D. Awschalom, and D. N. Basov, Phys. Rev. Lett. 89, 097203 (2002).

${ }^{33}$ A. Twardowski and C. Hermann, Phys. Rev. B 32, 8253 (1985).

${ }^{34}$ R. Braunstein and E. O. Kane, J. Phys. Chem. Solids 23, 1423 (1962).

${ }^{35}$ M. L. Huberman, A. Ksendzov, A. Larsson, R. Terhune, and J. Maserjian, Phys. Rev. B 44, 1128 (1991).

${ }^{36}$ W. Songprakob, R. Zallen, D. V. Tsu, and W. K. Liu, J. Appl. Phys. 91, 171 (2002).

${ }^{37}$ T. Schallenberg and H. Munekata, Appl. Phys. Lett. 89, 042507 (2006).

${ }^{38}$ S. Lee, A. Trionfi, T. Schallenberg, H. Munekata, and D. Natelson, Appl. Phys. Lett. 90, 032105 (2007).

${ }^{39}$ S. Ohya, H. Kobayashi, and M. Tanaka, Appl. Phys. Lett. 83, 2175 (2003).

${ }^{40}$ Jong Su Kim et al., J. Korean Phys. Soc. 39, S518 (2001).

${ }^{41}$ R. W. Glew, J. Cryst. Growth 68, 44 (1984).

${ }^{42}$ T. Yamada, E. Tokumitsu, K. Saito, T. Akatsuka, M. Miyauchi, M. Konagai, and K. Takahashi, J. Cryst. Growth 95, 145 (1989).

${ }^{43}$ T. Jungwirth, M. Abolfath, J. Sinova, J. Kučera, and A. H. MacDonald, Appl. Phys. Lett. 81, 4029 (2002).

${ }^{44}$ J. Sinova, T. Jungwirth, S. R. Eric Yang, J. Kučera, and A. H. MacDonald, Phys. Rev. B 66, 041202(R) (2002).

${ }^{45}$ S. R. Eric Yang, J. Sinova, T. Jungwirth, Y. P. Shim, and A. H. MacDonald, Phys. Rev. B 67, 045205 (2003).

${ }^{46}$ R. Moriya and H. Munekata, J. Appl. Phys. 93, 4603 (2003).

${ }^{47}$ J. D. Parsons and F. G. Krajenbrink, J. Electrochem. Soc. 130, 1782 (1983).

${ }^{48}$ Y. Nagai, T. Junimoto, K. Nagasaka, H. Nojiri, M. Motokawa, F. Matsujura, T. Dietl, and H. Ohno, Jpn. J. Appl. Phys., Part 1 40, 6231 (2001).

${ }^{49}$ K. Hirakawa, S. Katsumoto, T. Hayashi, Y. Hashimoto, and Y. Iye, Phys. Rev. B 65, 193312 (2002).

${ }^{50}$ E. J. Singley, K. S. Burch, R. Kawakami, J. Stephens, D. D. Awschalom, and D. N. Basov, Phys. Rev. B 68, 165204 (2003). 